\title{
Auch an das familiäre Mittelmeerfieber denken
}

Bei der Abklärung von unklarem Fieber sollte man nicht nur an die häufigsten Ursachen wie Infektionen, Neoplasien und Kollagenosen sondern auch an periodische Fiebersyndrome wie das familiäre Mittelmeerfieber denken.

Definitionsgemäß spricht man von einem Fieber unklarer Genese, wenn folgende Kriterien vorliegen:

- Krankheitsdauer länger als drei Wochen

- Fieber mehrmals über $38,3^{\circ} \mathrm{C}$

- Diagnose ist auch nach einwöchiger stationärer Abklärung unklar

„An den differentialdiagnostischen Überlegungen hat sich in den letzten hundert Jahren grundsätzlich nicht viel geändert", so Dr. Sebastian Jonas Saur vom Rheumazentrum der Universitätsklinik Tübingen beim virtuellen Satelliten-Symposium „Immer wieder Fieber? - Wenn die Ursache im Verborgenen liegt", im Rahmen des 127. Kongresses der Deutschen Gesellschaft für Innere Medizin (DGIM). Am häufigsten sind Infektionen, gefolgt von Neoplasien und Kollagenosen. Seltener sind die Sarkoidose, die Lungenembolie und die Polymyalgia rheumatica.

Wenn diese Ursachen ausgeschlossen sind, sollte man immer an ein Autoinflammationssyndrom denken. Bei Autoinflammationssyndromen handelt es sich um sehr seltene Erkrankungen. „Es vergehen oft viele Jahre, bevor die richtige Diagnose gestellt wird" so Saur. Dazu gehören die systemische juvenile idiopathische Arthritis (SJIA), auch Morbus Still genannt, und die periodischen Fiebersyndrome. (• Abb. 1)

\section{Übeltäter Interleukin-1 $\beta$}

Bei den genetisch determinierten periodischen Fiebersyndromen finden sich zusätzlich Hautausschläge und Schmerzen im Bauch- und Brustbereich. "Nicht selten manifestiert sich die Erkrankung als akutes Abdomen", so Privatdozent Dr. Arnd Giese, Chefarzt der internistischen Abteilung am St. Elisabeth-Hospital in Herten. Zu den periodischen Fiebersyndromen gehört neben anderen Formen (CAPS, HIDS, TRAPS) auch das Familiäre Mittelmeerfieber, das überwiegend bei Menschen aus der östlichen Mittelmeerregion auftritt. Es handelt sich um eine autosomal-rezessiv vererbte Erkrankung, sodass die Diagnose durch eine genetische Untersuchung gesichert wird.

Beider Pathogenese der Autoinflammationssyndrome spielt Interleukin- $1 \beta$ die entscheidende Rolle. Daraus ergibt sich als Therapieziel die Blockade von Interleukin-1 $\beta$ mit dem monoklonalen Antikörper Canakinumab. Eingesetzt werden sollte diese Substanz beim FMF dann, wenn die primäre Therapie mit
Colchizin nicht ausreichend wirksam ist oder nicht vertragen wird.

Bericht:Dr. Peter Stiefelhagen

Hinweis des Verlags. Der Verlag bleibt in Hinblick auf geografische Zuordnungen und Gebietsbezeichnungen in veröffentlichten Karten und Institutsadressen neutral.

rheuma plus $2021 \cdot 20: 107$

https://doi.org/10.1007/s12688-02100437-8

(c) Springer-Verlag GmbH Austria, ein Teil von Springer Nature 2021
Quelle:Virtuelles Satelliten-Symposium „Immer wieder Fieber? - Wenn die Ursache im Verborgenen liegt", im Rahmen des 127. Kongresses der Deutschen Gesellschaft für Innere Medizin (DGIM), 17.04.2021, Veranstalter: Novartis AG

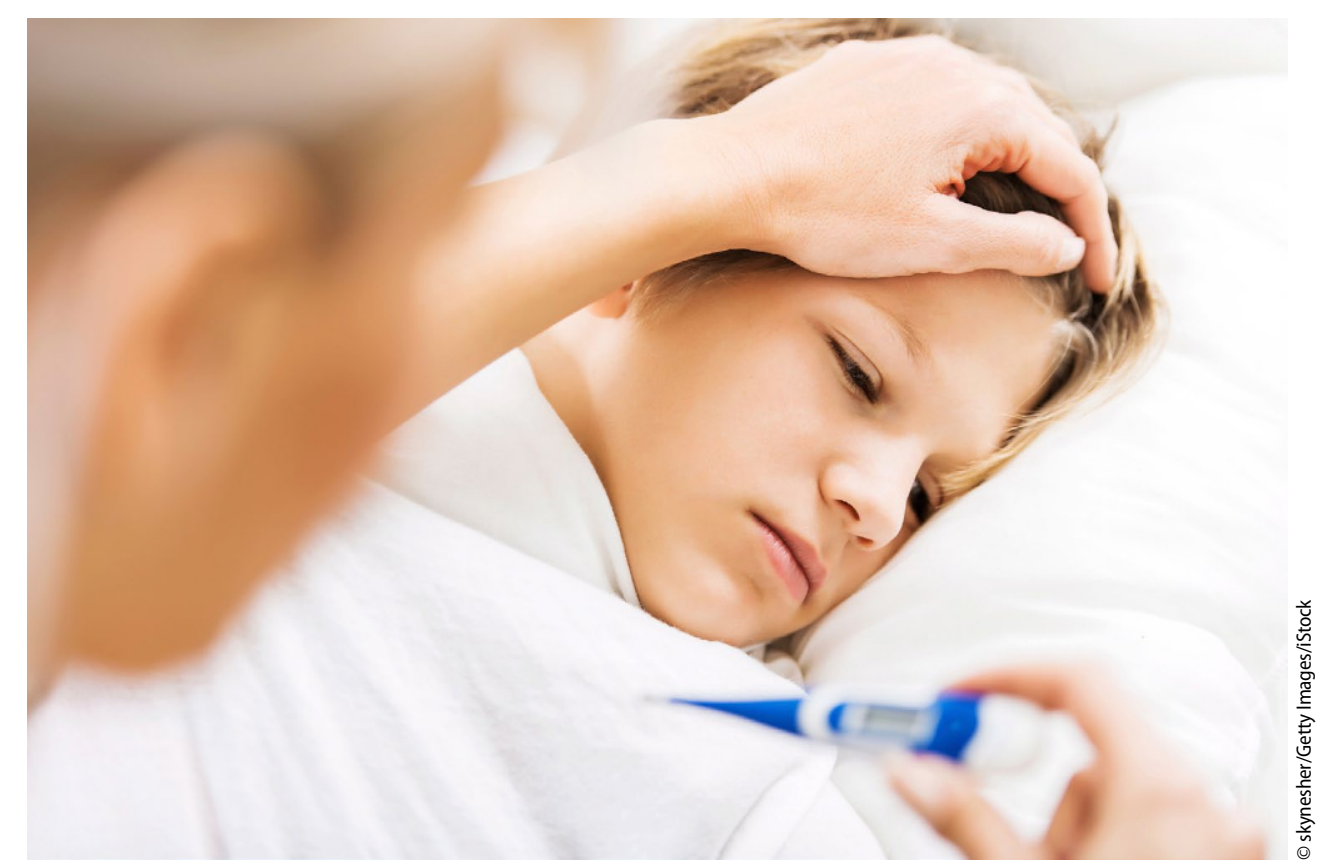

Abb. 1 \ Bei Autoinflammationssyndromen vergehen oft viele Jahre, bevor die richtige Diagnose gestellt wird. (Symbolbild mit Fotomodell) 